\title{
On the colour-colour properties of the Extremely Red Objects
}

\author{
S. Bergström ${ }^{1}$ and T. Wiklind ${ }^{2}$ \\ ${ }^{1}$ Department of Astronomy and Astrophysics, Centre for Astrophysics and Space Science, Chalmers University of Technology, \\ 43992 Onsala, Sweden \\ e-mail: stefan@oso.chalmers.se \\ 2 European Space Agency, Space Telescope Science Institute, 3700 San Martin Drive, Baltimore, MD 21218, USA \\ e-mail: wiklind@stsci.edu
}

Received 7 May 2003 / Accepted 10 October 2003

\begin{abstract}
The colours of the galaxy class known as Extremely Red Objects (EROs; $R-K>5$ ) are considered to be consistent with two distinct galaxy populations at high redshift: evolved ellipticals or young dusty starbursts. In this paper the properties of EROs, spanned by the five photometric bands RIJHK, are investigated as to the possibility to distinguish between these two galaxy populations using only broad band photometry. The broad band colours of elliptical and starburst galaxies at redshifts up to 5 are computed from synthetic spectra obtained using the spectral evolution synthesis programme PÉGASE.2. Two initial mass functions and a range of metallicities and extinctions are used. In order to be extremely red in the redshift range considered, the evolution of the $R-K$ colour sets the requirement that ellipticals have to be less than 7-8 Gyr old, and that the starbursts must have colour excesses of $E(B-V)>1$, as derived from the nebular emission lines. In investigating the overlap in the different colour-colour planes as a function of redshift, it is found that the planes formed from permutations of the same three filters exhibit very similar overlap characteristics. In colour-colour planes formed within such triplets one of the filters will serve as a "pivot" band against which the two other bands are compared. The configuration where this pivot band lies between the other two bands has the best performance as a discriminator among the three possible configurations. A consistent behaviour cannot be found among the configurations formed by permuting four filters. The minimal filter configuration $R-H$ vs. $H-K$ is found to be the very best discriminator, working as such up to redshift 2.9.
\end{abstract}

Key words. galaxies: elliptical and lenticular, $\mathrm{cD}$ - galaxies: evolution - galaxies: fundamental parameters galaxies: high-redshift - galaxies: starburst

\section{Introduction}

The optically faint high-redshift galaxy class known as Extremely Red Objects, or EROs, has since its discovery (Elston et al. 1988) been extensively studied. The EROs are considered to be one of the more enigmatic galaxy classes since their faintness makes it very difficult to classify them, either spectroscopically or morphologically. Although their extremely red colours, $R-K>5$, narrow down the possibilities to essentially ellipticals and dusty starburst galaxies at high redshifts, the colour degeneracy between these two very different galaxy populations prevents the determination of their morphologic types. Understanding the nature of the EROs is of great importance since they can provide a direct observational test of theories of galaxy formation (e.g. Cimatti 2001): the hierarchical clustering picture (e.g. White \& Frenk 1991; Kauffmann et al. 1993) versus the monolithical collapse picture (e.g. Eggen et al. 1962; Larson 1975).

Send offprint requests to: $\mathrm{S}$. Bergström, e-mail: stefan@oso.chalmers.se
Due to their faintness, only a handful EROs have reliable redshift estimates in combination with a determination of the morphologic type. These individual cases divide evenly between starbursts (e.g. Cimatti et al. 1998; Dey et al. 1999; Afonso et al. 2001; Smith et al. 2001; Frayer et al. 2003) and ellipticals (e.g. Spinrad et al. 1997; Soifer et al. 1999; Stiavelli et al. 1999; Saracco et al. 2003), and do not allow any conclusion to be drawn as to what galaxy type the majority of the EROs belongs to. The individually studied elliptical EROs are located in a narrow redshift range, $1.2<z<1.8$, whereas the individually classified starburst systems span a much larger redshift range.

Surveys have shown that the EROs comprise a heterogeneous galaxy population (Thompson et al. 1999; Cimatti et al. 1999; Moriondo et al. 2000), although the elliptical case has been the stronger one supported by in particular the detected clustering of the EROs (Daddi et al. 2000; Moustakas \& Somerville 2002). However, the sample of EROs compiled by Daddi et al. (2002) contains similar fractions of star-forming and evolved galaxies, with only the evolved galaxies showing strong clustering. In later surveys, the heterogeneity of the 
ERO population has been confirmed (e.g. Mannucci et al. 2002; Smail et al. 2002; Smith et al. 2002a; Moustakas et al. 2003; Yan \& Thompson 2003) and the picture is further complicated by the finding by Smith et al. (2002b) of an early-type disc ERO at $z=1.6$.

Taking the properties of the ERO sample (complete to $\left.K_{\mathrm{s}} \leq 19.2\right)$ obtained by Cimatti et al. (2002) as "typical", the ERO population consists of similar fractions of "old" and "starforming" galaxies. The average "old" ERO is concluded to be at least 3 Gyr old, being formed beyond $z=2.4$, if the metallicity is solar. The average "star-forming" ERO agrees with a starburst spectrum having a Calzetti et al. (2000) $E(B-V)_{\text {star }}$ of $\sim 0.8$, i.e. an extinction for the nebular emission lines of $E(B-V)=1.8$. If the contribution from an assumed underlying old stellar population is accounted for, the average reddening can be decreased to $E(B-V)_{\text {star }} \sim 0.7$. Using other synthetic spectra, the extinction is found to be in the range $0.6<E(B-V)_{\text {star }}<1.1$.

Since EROs are by their definition very weak at optical wavelengths, spectroscopy is not a viable method for classifying large numbers of EROs. Instead, attempts have been made to use broad band colours. Pozzetti \& Mannucci (2000, hereafter PM00) found that EROs in the redshift ranges $1 \leq z \leq 2$ and $2 \leq z \leq 2.5$ fall in different regions in both $I-K$ vs. $J-K$ and $R-K$ vs. $J-K$ plots for sources in the lower redshift range, and in $H-K$ vs. $J-K$ plots for sources in the higher redshift range. This result is robust and valid for a variety of metallicities, dust contents, star formation histories and redshifts of formation.

Pierini et al. (2003) recently extended the study of the colour-colour method for classifying EROs. The study is similar to that of PM00 but includes dusty post-starburst galaxies as well as the nominal dust-free evolved stellar populations (old ellipticals) and dusty starbursts. Pierini et al. also studied the impact of various dust distributions within the model galaxies. While the dust distribution can change the attenuation properties, it did not have a large impact on the overall results, which were similar to those of PM00. The presence of dusty poststarburst systems tends to make the separation of old, dust-free systems and dusty starburst systems less distinct.

In this work, the possibility to use broad band colours to distinguish between the elliptical and dusty starburst galaxy populations among the EROs at redshifts below five is further pursued, and all possible colour-colour configurations of the five broad band filters $R I J H K$ are investigated. The study is based on models of the elliptical and dusty starburst populations, where synthetic galaxy spectra are obtained using the spectral evolution synthesis programme PÉGASE.2 (Fioc \& Rocca-Volmerange 1997, 1999). In particular, colour-colour combinations are searched for where the two galaxy populations do not overlap over a large redshift range. The intention is to study to what extent photometric classifications of EROs can be used. The adopted cosmology is $H_{0}=70 \mathrm{~km} \mathrm{~s}^{-1} \mathrm{Mpc}^{-1}$, $\Omega_{\mathrm{m}}=0.3$ and $\Omega_{\Lambda}=0.7$, thus yielding an age of the Universe of $13.5 \mathrm{Gyr}$.

The paper is organised as follows. The properties of the different galaxy models are presented in Sect. 2. In Sect. 3 the methods used are accounted for, which include determining which models produce EROs considering redshift and galaxy age, and investigating the overlap between the colours of the galaxy populations in different colour-colour diagrams. The results are presented and discussed in Sect. 4, and Sect. 5 concludes.

\section{The galaxy models}

In general there are two distinct galaxy populations that the EROs are considered to belong to: ellipticals and dusty starbursts. The elliptical galaxies consist of an old stellar population and have none or small amounts of gas and dust. Hence the colours of these galaxies are determined by the old stellar population which produce a large $4000 \AA$ break in the spectral energy distribution (SED). The colours of ellipticals can therefore be very red, especially if the galaxies are located at high redshift. The other galaxy population producing EROs is the dusty starbursts. These galaxies contain much gas and dust and have high star formation rates (SFRs). The light of the early stellar population is scattered and absorbed by the dust, which heats up and subsequently re-radiates the energy in the farinfrared. Since this extinction is greater at shorter wavelengths, the initially blue SED is reddened. Furthermore, a redshifted SED will be redder than the restframe counterpart.

To model these galaxy populations, the spectral synthesis evolution programme PÉGASE. $2^{1}$ (Fioc \& Rocca-Volmerange 1997, 1999) is used. The programme computes models of galactic spectra in the range $91 \AA$ to $160 \mu \mathrm{m}$ assuming different star formation scenarios, metallicities etc. PÉGASE. 2 uses evolutionary tracks of mainly the Padova group (see Fioc \& Rocca-Volmerange 1999 for details), and synthetic spectra are used when observational libraries are not available. For the nebular emission, only case B recombination is taken into account when computing the hydrogen line fluxes. For other lines, typical observed $\mathrm{H} \beta$ ratios are used. The programme allows for a consistent metallicity evolution using the Woosley \& Weaver (1995) models of SNII ejecta.

The elliptical galaxies are modelled as galaxies that after an instantaneous burst of star formation evolve passively, also known as a simple stellar population (SSP) model. (This is the (instantaneous-)burst population of Bruzual \& Charlot 1991, 1993.) After the burst of star formation the galaxies are assumed to contain no interstellar gas or dust, which is equivalent to assuming the on-set of a strong galactic wind. The galaxies are considered as ellipticals $100 \mathrm{Myr}$ after the starburst, and are then let to evolve for a Hubble time. The time grid consists of 128 epochs with a spacing of about $5 \%$ of the galaxy age.

The starburst galaxies are modelled as galaxies with a constant star formation during $100 \mathrm{Myr}$. The time step is $1 \mathrm{Myr}$. The extinction in the starbursts is modelled as a uniform screen of dust and gas and follows the Calzetti et al. (2000, hereafter C00) obscuration law derived for starbursts. According to the work by $\mathrm{C} 00$, the stellar continuum and the nebular emission lines suffer from different extinctions, due to the different stellar environments. The extinction of the stellar continuum is

${ }^{1}$ PÉGASE. 2 is made publicly available at the web site http://www.iap.fr/pegase/ 
proportional to the extinction of the nebular emission lines as given by the empirically derived relation

$E(B-V)_{\text {stars }}=0.44 E(B-V)_{\text {gas }}$.

Throughout this paper the extinction will be parameterised using the colour excess of the nebular emission lines, and the subscript gas is dropped (e.g. C00).

The C00 extinction law expresses the selective absorption, not total. The shape of the extinction law depends on the properties of the dust grains causing the extinction, whereas the amount of extinction can vary significantly. Here, the extinction is treated as a screen through which the radiation from the galaxies passes, and the amount of extinction can be varied by increasing the thickness of the screen. The colour excess required to produce an ERO of a particular galaxy at a given redshift is therefore only indicative of the amount of extinction needed. The total column density of dust, for example, will be dependent on the geometry of the stars and the obscuring medium.

The choice of SFR in the starbursts requires some consideration as it influences the SED in two ways. (i) The SFR provides a scaling factor of the flux of the spectrum. This scaling property does not have any impact on the colours as these are derived from the shape of the spectrum, not its absolute value. This is true also in the presence of nebular emission lines, as the continuum flux and emission line flux (through the flux of ionising photons) both are proportional to the number of stars produced. This has the result that two galaxies of the same metallicity, IMF and temporal shape of the star formation history will have the same colours regardless of the magnitude of the SFR. (ii) The SFR will influence the rate at which the galaxies are enriched: the higher the SFR, the faster the enrichment. This will cause a major change in the colours. Hence, of these two effects it is only the latter, where the metallicity is increased, that has a significant effect on the colours.

Since a consistent evolution of the metallicity is used in the modelling of the galaxy spectra, the metallicities will change in a galaxy experiencing an extended period of star formation (i.e. the starburst model). Following the line of reasoning sketched out in the previous paragraph, the SFRs cannot be too high if the range of metallicities is to be kept in the modelling. Hence, the value of the SFR is chosen to achieve a well controlled range of metallicities. The metallicity grid consists of five metallicities, $0.02,0.2,0.4,1$ and $2.5 Z_{\odot}$, which in the passive evolution of the ellipticals do not change, but in the case of the starbursts, these are initial values.

The SFR chosen to ensure such a slow enrichment is $5 \times 10^{-11} M_{\text {galaxy }} \mathrm{yr}^{-1}$. A galaxy with such an SFR would not be regarded as a starburst galaxy since only $0.5 \%$ of the gas mass have been used to form stars after $100 \mathrm{Myr}$. However, since the magnitude of the SFR does not influence the colours of galaxies of the same metallicity and stellar population, as explained above, this low-SFR model is equivalent to models having a more "realistic" SFR. Considering the metallicity in the ISM, an initial solar metallicity has been increased to $1.0050 Z_{\odot}$, i.e. by $0.5 \%$, after $100 \mathrm{Myr}$ for a starburst of Salpeter IMF. The enrichment is stronger the lower the initial metallicity, and the

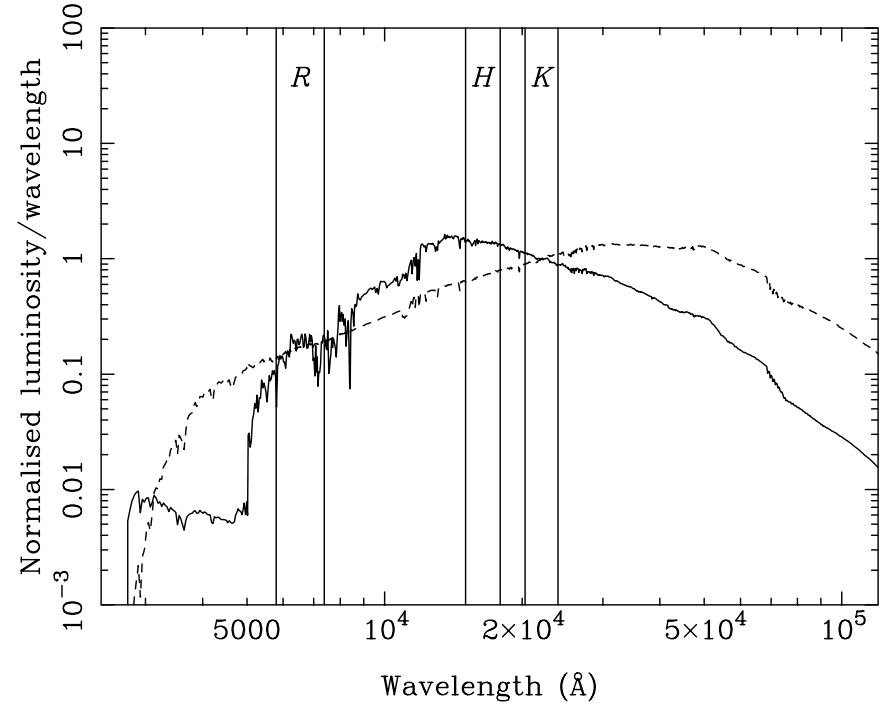

Fig. 1. Spectral energy distributions of galaxies at $z=2$ having Salpeter IMF and solar metallicity, normalised in the $K$ band. The solid line is the SED of a 1 Gyr old elliptical galaxy; the dashed line is the SED of a $100 \mathrm{Myr}$ old starburst galaxy having $E(B-V)=2$. Shown are also the wavelength regions covered by the $R, H$ and $K$ filters.

highest increase of metallicity occurs for the starbursts of initial metallicity $0.02 Z_{\odot}$ and Salpeter IMF; after $100 \mathrm{Myr}$, the metallicity have increased by $\sim 25 \%$.

The initial mass functions (IMFs) used in the modelling are the Salpeter (1955) and the "present-day" Kroupa (2001, Eq. (6)) IMFs in the mass range $0.09 \leq M / M_{\odot} \leq 120$. The Kroupa IMF is mainly based on the power-law compilation by Scalo (1998) but the effects of unresolved binaries are also taken into account. This IMF differs from the Salpeter IMF below $1 M_{\odot}$ and is included to increase the generality of the results. The adopted mass limits are consistent with the stellar tracks used by PÉGASE.2. The parameters common to all the models are shown in Table 1.

Model spectra of a 1 Gyr old elliptical and a $100 \mathrm{Myr}$ old starburst galaxy of $E(B-V)=2$ are shown in Fig. 1. The galaxies have Salpeter IMF and solar metallicity. Inlaid are the wavelength regions of the $R, H$ and $K$ bands. In Fig. 2, spectra of galaxies of ellipticals 200, 1000 and 5000 Myr old (left panel) and of starbursts 0,10 and 100 Myr old having $E(B-V)=2$ (right panel) are shown.

\section{The method}

Two questions are addressed in the present work: (i) When can ellipticals and starbursts be EROs? (ii) Is it possible to distinguish between the extremely red ellipticals and dusty starbursts merely from their broad band colours? A first attempt to answer the second question was made by Scodeggio \& Silva (2000). In a similar study, PM00 were able to separate old elliptical galaxies from dusty starbursts using $I-K$ vs. $J-K$ and $R-K$ vs. $J-K$ plots in the redshift range $1 \leq z \leq 2$, and also $H-K$ vs. $J-K$ in the range $2 \leq z \leq 2.5$. 

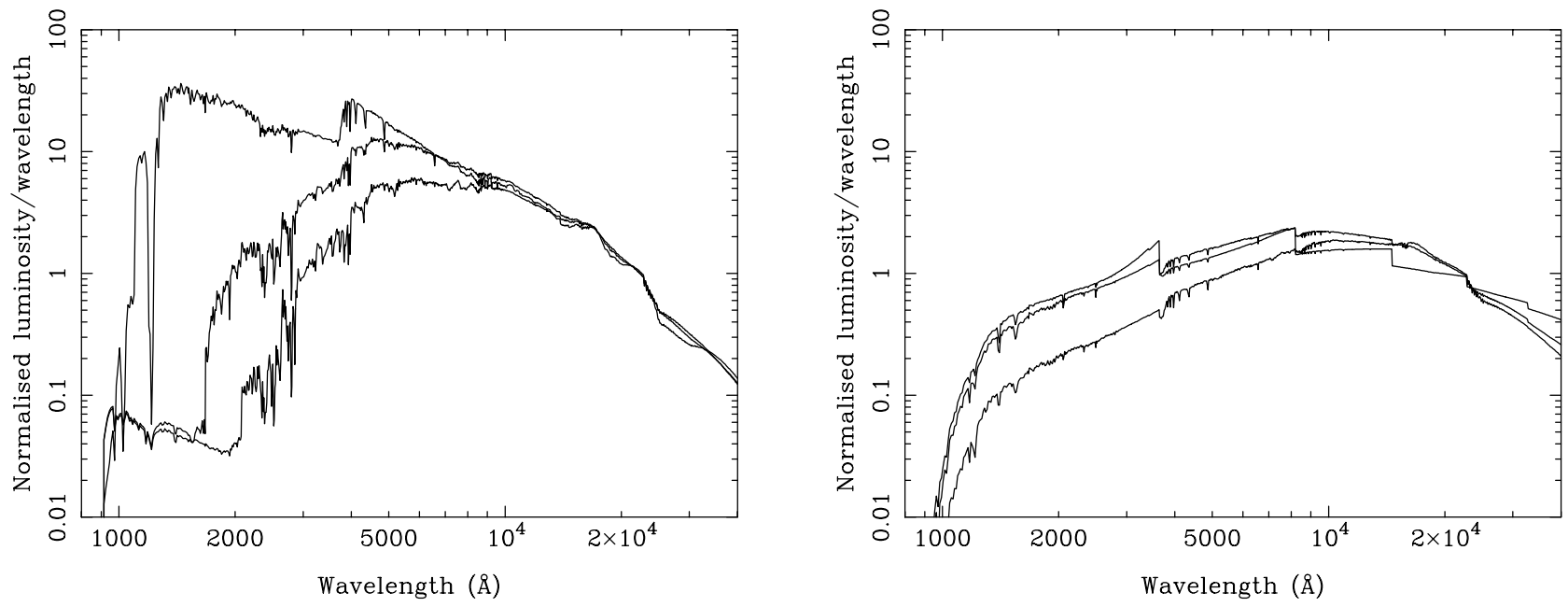

Fig. 2. Spectral energy distributions of galaxies having Salpeter IMF and solar metallicity, normalised in the $K$ band. Left panel: a $200 \mathrm{Myr}, 1$ Gyr and $5 \mathrm{Gyr}$ old elliptical. Right panel: a new-born, 10 and $100 \mathrm{Myr}$ old starburst galaxy having $E(B-V)=2$. Both galaxy types become redder with age.

Table 1. PÉGASE.2 parameters common to all the models.

\begin{tabular}{ll}
\hline \hline Property & Value \\
\hline Supernova ejecta & $\begin{array}{c}\text { Woosley \& Weaver (1995) } \\
\text { model B }\end{array}$ \\
$\begin{array}{l}\text { Infall } \\
\text { Stellar winds }\end{array}$ & Yes \\
$\begin{array}{l}\text { Fraction of close } \\
\text { binary systems }\end{array}$ & 0.05 \\
$\begin{array}{l}\text { Metallicity evolution } \\
\text { Formation of substellar } \\
\quad \text { objects }\end{array}$ & Consistent \\
Nebular emission & No \\
Extinction & Yes \\
\hline
\end{tabular}

In the work presented here, the ability to distinguish between the considered galaxy types are investigated for all the 45 possible combinations of the RIJHK filters and for all plausible redshift ranges. This section describes the methods used to investigate the colour evolution and the colour plane properties.

The fluxes at different redshifts are computed from the spectra obtained from PÉGASE. 2 using the standard relation

$F\left(\lambda_{0}\right)=\frac{L\left(\lambda_{1}\right)(1+z)}{4 \pi D_{\mathrm{L}}^{2}}$,

where $\lambda_{0}=(1+z) \lambda_{1}$ and $D_{\mathrm{L}}$ is the luminosity distance. The magnitudes are then computed in the Vega system using the well-documented filter functions of Bessell \& Brett (1988) and Bessell (1990) founded on the Cousins system. The filter response functions are extended to 1500 data points using cubic splines. This filter system is very similar to the filters used in the EIS-Deep Survey, the SUSI RI and SOFI JHK filters at the $\mathrm{NTT}^{2}$, so any conclusions made in the following will not be altered significantly if the latter system is used.

2 Filter functions achieved from the Filter Catalogue at the ESO La Silla home page, http://www.1s.eso.org
Table 2. The properties of the normalised (unity peak transmission) Bessell filters. The width is defined as the area of the filter response function.

\begin{tabular}{lcll}
\hline \hline Band & $\lambda_{\text {mean }}(\AA)$ & Width $(\AA)$ & $F_{0}\left(\mathrm{~W} \mathrm{~m}^{-2} \AA^{-1}\right)$ \\
\hline$R$ & 6586 & 1581 & $2.216 \times 10^{-12}$ \\
$I$ & 8060 & 1495 & $1.154 \times 10^{-12}$ \\
$J$ & 12368 & 2023 & $3.256 \times 10^{-13}$ \\
$H$ & 16466 & 2855 & $1.181 \times 10^{-13}$ \\
$K$ & 22119 & 3660 & $4.110 \times 10^{-14}$ \\
\hline
\end{tabular}

\subsection{Colour evolution}

Two approaches are used to find galaxies that might produce EROs under certain circumstances. The first approach is to find the lowest redshift required to make a galaxy of a certain age extremely red. The redshift where $R-K=5$ will hereafter be called $z_{\text {ERO }}$. This procedure imposes a cosmology consistency criterion: the redshift of formation, $z_{\mathrm{f}}$, of a galaxy observed (as an ERO for instance) at a certain redshift has to be consistent with the assumed cosmology. As will be seen, this cosmological constraint rules out that ellipticals capable of being classified as EROs are older than about 7-8 Gyr.

The second approach is to study how the $R-K$ colour evolves with redshift. This can be done both by assuming a redshift of formation, $z_{\mathrm{f}}$, and let a galaxy evolve in time, or by redshifting a galaxy of a fixed age.

\subsection{Overlap in the colour plane}

The main aim of this work is to investigate whether broad band colour-colour plots can be used to discriminate between the two main classes of galaxies making up the ERO population: evolved ellipticals and dusty starbursts. The colours of elliptical and starburst galaxies at different redshifts may or may not overlap, and there could exist redshift ranges in which the colour-colour regions of the two galaxy populations can be 
distinctly defined. To investigate this, the first step is to build up a grid of colours of ERO-classified galaxies. For a range of redshifts, the colours of ellipticals and starbursts are computed. After removing those that are neither consistent with the cosmology nor have $R-K \geq 5$, two sets of colour-colour points are obtained, associated with the EROs in each galaxy population.

The overlap that occurs in a colour-colour diagram between the colours of the two galaxy populations at different redshifts is then computed. The confusion, or overlap, between these two point sets are defined as follows. If $A$ designates the region covered by the one set, and $B$ the region of the other set, then the intersection of these two sets, $A \cap B$, is the region where the two sets overlap. The measure of overlap is now taken as the area of the intersection normalised by the area of the smallest region, i.e.

overlap $=\frac{A \cap B}{\min \{A, B\}}=\max \left\{\frac{A \cap B}{A}, \frac{A \cap B}{B}\right\}$.

In this way, an overlap measure of 1 is obtained if one of the sets is completely embedded in the other.

To define the regions covered by the point sets, every colour-colour point is considered as defining the centre of a circle having a fixed (small) radius. If the point density is high enough, this will create a continuous region. Two points are now regarded as overlapping if the distance between them is less than twice the radii of the circles. This requires that the point density is homogeneous, since it cannot be accepted that a higher point density part of the colour-colour region will have a higher weighting in the overlap computation. Hence, the two point sets are discretised into an evenly spaced grid, with a spacing of one circle radius. The colour-colour points will be offset from their true loci by at most one half of a circle radius. In the overlap computation, the circle radius is set to $0.05 \mathrm{mag}$, which will make two points more than 0.1 mag apart seen as separate. The gridding will shift the colour-colour points by at most 0.025 mag, which is well below the typical photometric errors.

This procedure results in maps of overlap measures where one of the axes corresponds to the redshifts of the ellipticals, and the other to the redshifts of the starbursts (see Fig. 6). From these maps, the redshift regions where the starburst EROs and the elliptical EROs can be separated from each other in a colour-colour diagram can be clearly seen. What is left is to explore these redshift regions to see what colours the different galaxies have.

\section{Results and discussion}

\subsection{Colour evolution}

\subsubsection{The ellipticals}

All ellipticals have $R-K>5$ at some redshift and the general trend of the $R-K$ colour is to increase with redshift. The explanation for this can be seen in the left panel of Fig. 2: when the SED of an elliptical is redshifted, it will be shifted to the right in the diagram, and except for the youngest ellipticals, the $R$ filter will soon measure in the less luminous region blue-ward of the $4000 \AA$ break, while the $K$ filter measures brighter and brighter

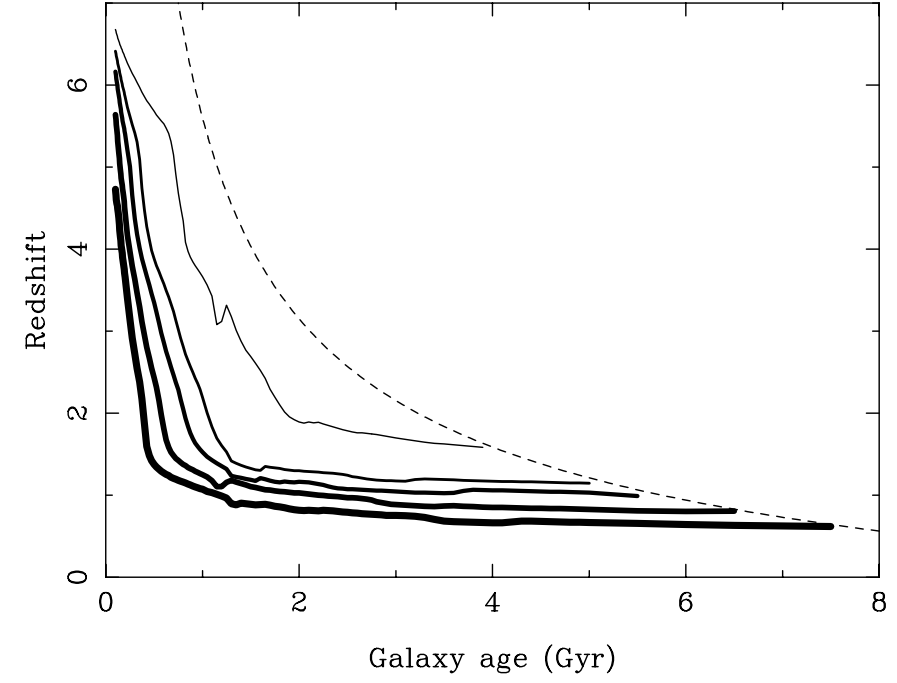

Fig. 3. $z_{\text {ERO }}$ vs. galaxy age for the ellipticals of Salpeter IMF, where $z_{\text {ERO }}$ is defined as the lowest redshift where an elliptical can be an ERO. The metallicities $\left(0.02-2.5 Z_{\odot}\right)$ of the models increase downwards/leftwards and the lines thicken accordingly. The dashed line traces the cosmological redshift-age relation.

parts of the SED. A higher metallicity reddens the SED due to line-blanketing and backwarming in the stellar atmospheres. A redder galaxy will require a lower redshift to make the galaxy extremely red, i.e. a lower $z_{\text {ERO }}$ (as defined in Sect. 3.1). As a result of this, an elliptical of higher metallicity can be encountered as an ERO over a larger redshift range and hence it can also be older compared to an ERO elliptical of lower metallicity.

In Fig. 3 the $z_{\mathrm{ERO}} \mathrm{s}$ for the ellipticals are plotted as a function of galaxy age. The dashed line shows the redshiftage correspondence for the chosen cosmology: the area leftwards/downwards of this line contains the redshift-age set consistent with the cosmology. The five solid lines show the $z_{\mathrm{ERO}} \mathrm{S}$ for ellipticals of different metallicities $\left(0.02-2.5 Z_{\odot}\right)$, and the thickness of the lines increases with increasing metallicity. Since $z_{\text {ERO }}$ is the lowest redshift needed to produce an ERO, the redshift regime between the "cosmology line" and each $z_{\text {ERO }}$ line shows the redshifts where the EROs exist. It is clearly seen that the cosmology requires the ellipticals to be younger than 7.5 Gyr. Furthermore, the lowest $z_{\text {ERO }}$ is about 0.7 , a result which is consistent with the observations of Moustakas et al. (2003), who find very few early-type EROs at redshifts below $\sim 0.9$.

The elliptical $R-K$ colour for different assumed redshifts of formation is shown in Fig. 4. The lowest metallicity, $Z=$ $0.02 Z_{\odot}$, only produces EROs in a small redshift interval around $z=2$ for the highest $z_{\mathrm{f}}$. For decreasing $z_{\mathrm{f}}$, the limiting EROproducing metallicity increases, and when $z_{\mathrm{f}}=1$, not even the highest metallicity will yield red enough galaxies.

\subsubsection{The starbursts}

For starburst galaxies, the evolution of the $R-K$ colour with redshift puts constraints on the amount of reddening needed 

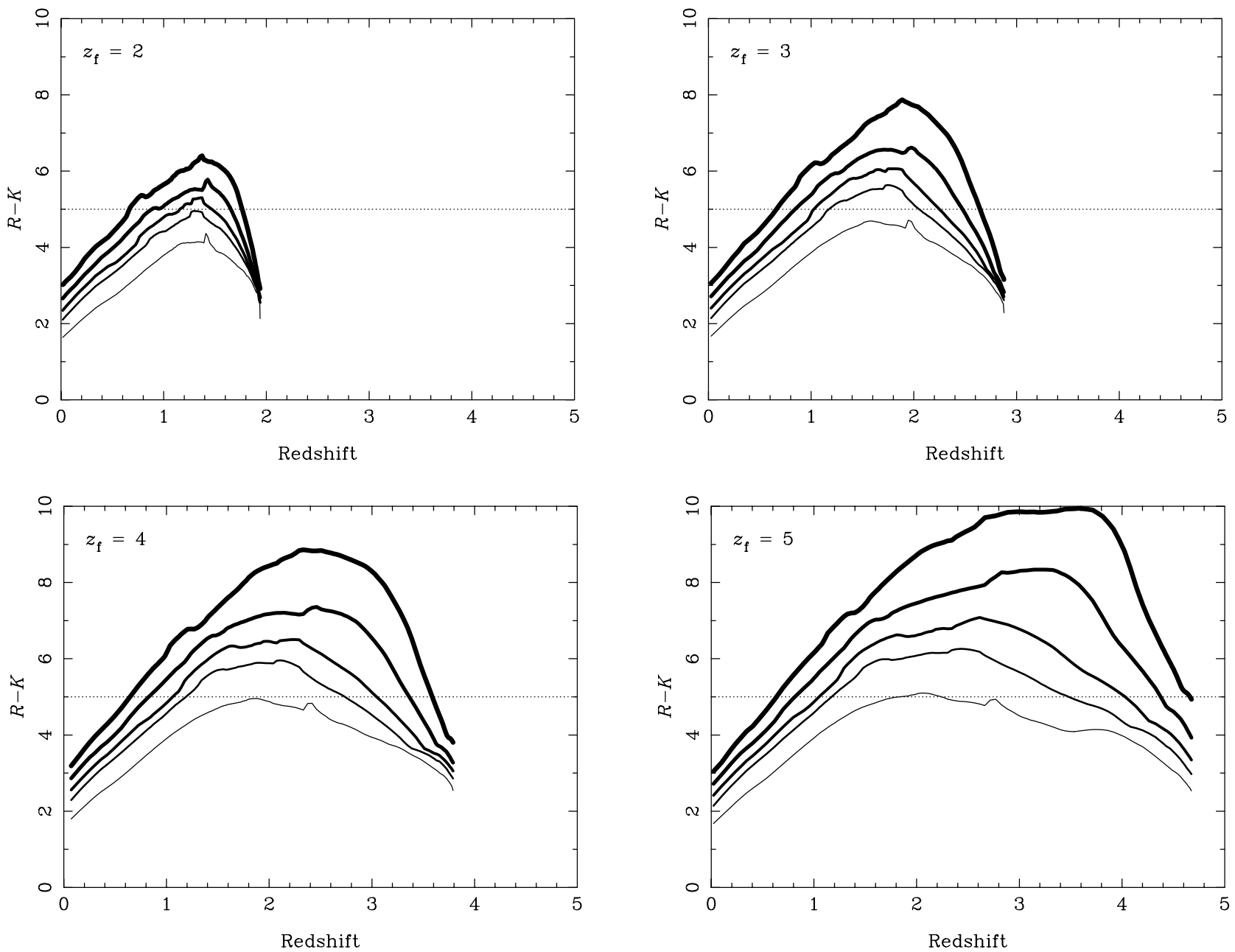

Fig. 4. Colour-redshift diagrams for the ellipticals of Salpeter IMF assuming formation redshifts from 2 to 5 using the full range of metallicities $\left(0.02-2.5 Z_{\odot}\right)$. The thickness of the lines increases according to the metallicity.

to produce EROs. This limiting amount of extinction will decrease as the metallicity increases, since a more metal-rich galaxy will be redder. The colour-redshift diagram in Fig. 5 shows the regions of the $R-K$ colour of the starbursts for colour excesses of 1.0 and 2.0. The full ranges of galaxy age $(0-100 \mathrm{Myr})$ and metallicity $\left(0.02-2.5 Z_{\odot}\right)$ are used. A colour excess of slightly more than 1 is required to produce EROs for $z \leq 5$. Excepting the very lowest redshifts, the starbursts can be observed as EROs at all $z \leq 5$ when $E(B-V)=2.0$.

\subsection{The colour plane properties}

The following investigation of the colour-colour space, both as the overlap maps and the colour-colour plots are concerned, does not depend on any assumed formation redshifts. The galaxies are assumed to form continuously, and only the cosmology sets the age limits for the galaxies. At a certain redshift the considered galaxy models are those that strictly fit into the current lifetime of the Universe. No assumption is made of a redshift where the galaxy formation is started in the Universe as a whole.

\subsubsection{The overlap in the colour-colour diagrams}

Distinguishing between the elliptical and starburst models in colour-colour diagrams is possible only if the colours of the galaxies follow evolutionary tracks that do not overlap. As a first test, colour-colour plots of all the 45 colour-colour configurations that can be formed from the filters RIJHK are made for the whole redshift grid (redshifts below 5 in steps of 0.1 ). Both Salpeter and Kroupa IMFs are used, and the colour excess of the starbursts ranges up to 2.0 in steps of 0.1 . It is found that when considering this broad redshift range, all filter configurations produce colour-colour plots where the region of the starburst colours lie almost completely inside the colour region of the ellipticals, hence they cannot be separated in this type of plots.

The next step is to see in which redshift ranges the different configurations produce colours where the galaxy populations are distinguishable, and the overlap is computed using the procedure described in Sect. 3.2. The model grid is again set up using Salpeter and Kroupa IMFs and colour excesses of the starbursts up to 2 in steps of 0.1 . The redshift grid spacing is 0.1 . Overlap maps resulting from this procedure are shown in Fig. 6. 


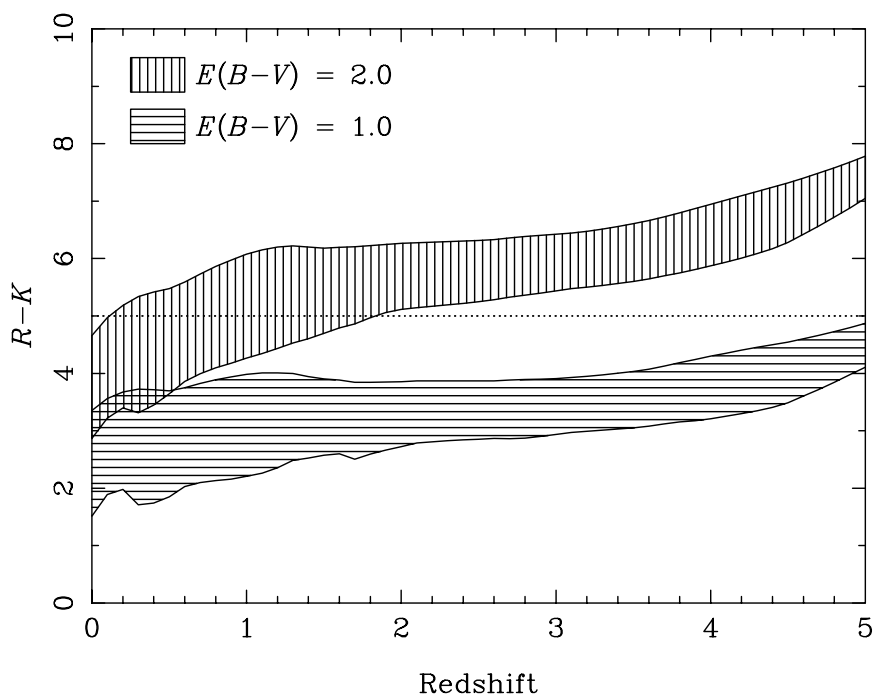

Fig. 5. Colour-redshift diagram for the starbursts of Salpeter IMF. The vertically hatched region corresponds to $E(B-V)=2.0$, and the horizontally hatched region to $E(B-V)=1.0$. The full age and metallicity ranges are used; the more metal-rich and the older the galaxy, the redder.

A proper interpretation of these maps requires some remarks. (i) The overlap has to be small over large continuous regions in the redshift space, starting from $z=0$ and up to high redshifts. A colour configuration is of course only useful as a discriminator at redshifts below the redshift where the colours starts to overlap. (ii) Since the fraction of EROs in a given galaxy population increases with redshift, the region occupied by EROs in a colour-colour plot will increase with redshift as well. At low redshifts, the colour region covered by the starbursts can therefore be very small, which will make any overlap with the ellipticals to appear more significant than what is reasonable. Hence it is not enough to decide on a redshift limit for a certain filter configuration from its overlap map, but to determine the impact of the overlap at low redshifts, the corresponding colour-colour diagram has to be investigated. (iii) The ridges in the overlap maps often represent cases where the colours of one galaxy population cross over the colour region of the other. This causes the colour-colour plots to exhibit very complicated structures which makes it difficult to define a separation line.

The configurations that can be formed from permutations of a set of three bands (e.g. the triplet $\{R H K\}$ from which the filter configurations $R H H K^{3}, R K H K$ and $R H R K$ may be formed) have a "pivot band" against which the two other magnitudes are compared, e.g. the $K$ band in the $R K H K$ configuration. The configurations formed from such a triplet are found to have very similar overlap features, although small differences can be seen. For all the triplets, the configurations where the pivot wavelength band lies between the other two bands, are always the best ones; for instance $R H H K$ in the triplet $\{R H K\}$. It should be noted that this result is consistently observed throughout the course of this work. The worst

\footnotetext{
3 The notation " $X Y Z W$ " will be used as an abbreviated form of " $X-Y$ vs. $Z-W "$.
}

performance is in most cases exhibited by the configurations using the shorter wavelength band as pivot band, although some (small) changes can be seen if higher extinctions are included.

The configurations formed from quadruples (configurations made with four different bands, e.g. the quadruple $\{R J H K\}$ from which the configurations $R J H K, R H J K$ and $R K J H$ may be formed) tend to behave very differently and cannot be regarded as forming distinct groups having similar overlap features. Since they are based on four regions on the SED, they are more sensitive to differences between the SEDs. However, in the overlap maps this advantage over the triplets manifests itself only in that the average performance of the quadruples is better than the triplets, but the best triplet has still a higher redshift limit than the best quadruple configuration. Furthermore, the only quadruple that actually performs better than the four triplets it can be broken into is the $\{I J H K\}$ quadruple. This combination of filters appears unfortunate; the triplets $\{I H K\}$, $\{J H K\}$ and $\{I J H\}$ are the three worst of the triplets, and the $\{I J K\}$ as well as $\{I J H K\}$ itself both yield complicated colourcolour plots. For the other quadruples, there is always a configuration of three filters that performs at least as well as its "parent" quadruple.

The relative discriminating performance of the three configurations within each filter set are shown in Table 3. Figure 6 shows the filter configuration yielding the best overlap properties within a given filter set. The colour coding means the brighter the area, the less overlap. The RHHK configuration has one of the highest redshift limits, and can discriminate between the galaxy populations up to redshift 2.9. The small overlap between ellipticals and starbursts at $z \sim 1$ for this configuration does not pose a problem. This will be discussed further in the next section.

\subsubsection{Non-overlapping colour regions}

Having found the redshift regimes where the best colourcolour plots exhibit distinct elliptical and starburst colour regions as described in Sect. 4.2.1, an attempt is now made to define lines that separate the two regions. A filter configuration can be considered as having an improved performance as a discriminator if the separation line is parallel with the reddening vector, since an increase in extinction will not cause the starbursts to cross such a separation line. However, as a higher extinction also will enable lower-redshift starbursts to become EROs, it is possible that these "new" candidates will intrude on the regions of the ellipticals.

A separation line between the colour regions of ellipticals and starbursts are defined for the configuration having the best performance within each filter set, see Table 3. For a given colour-colour configuration, it is in many cases possible to define a whole set of lines separating the two regions; in these cases a representative line is presented. The separation line is valid for $z \leq z_{\max }$, where $z_{\max }$ is the redshift limit beyond which the colours will be confused. A separation line is not defined for the filter sets that have redshift limits below about one, as nonoverlap at such low redshifts are due to a lack of EROs among the ellipticals and/or the starbursts. 

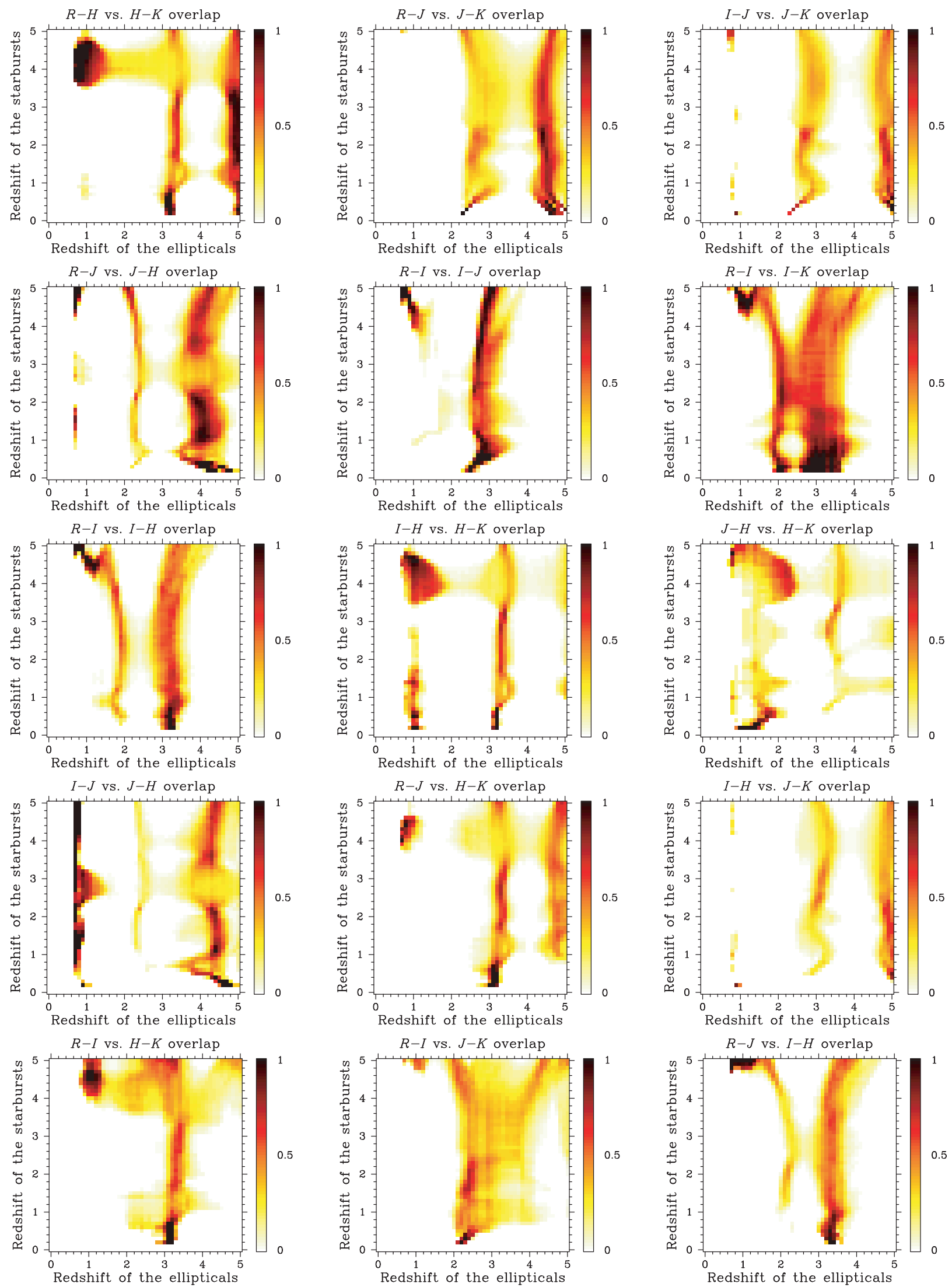

Fig. 6. The colour overlap maps for the filter configurations yielding the best discriminator performance within each filter set. The brighter the area, the less overlap; white corresponds to a separation of the elliptical and starburst EROs by at least 0.1 mag in the corresponding colourcolour plot. The galaxy models have Salpeter and Kroupa IMFs, metallicities from 0.02 to $2.5 Z_{\odot}$, and for the starbursts, colour excesses up to 2.0. Only models yielding EROs consistent with the cosmology are considered in computing the colour overlap. 
Table 3. This table shows (i) The relative performance of the filter configurations within each filter set. This is based on the ability to discriminate between the colours of the modelled ellipticals and starbursts for $E(B-V) \leq 2$. (ii) The separation line corresponding to the configuration that has the best discriminating performance within each filter set. (iii) The redshift limit beyond which the colour regions become entangled, $z_{\text {max }}$. The separation line is valid up to this redshift limit. Separation lines are not defined for the filter configurations having a redshift limit less than about one. (iv) The faintest ERO $K$ magnitude of $L^{*}$ ellipticals and starbursts at $z_{\max }$.

\begin{tabular}{|c|c|c|c|c|c|c|c|}
\hline \multirow[t]{2}{*}{ Filter set } & \multicolumn{3}{|c|}{ Configuration performance } & \multirow{2}{*}{$\begin{array}{l}\text { Separating line: colours of } \\
\text { ERO ellipticals at } z \leq z_{\max }\end{array}$} & \multirow[t]{2}{*}{$z_{\max }$} & \multicolumn{2}{|c|}{$K\left(L_{\text {bol }}=L^{*}\right)$ at $z_{\max }$} \\
\hline & Best & $\longrightarrow$ & Worst & & & Ellipticals & Starbursts \\
\hline$\{R H K\}$ & RHHK & RKHK & RHRK & $(R-H)>3.69(H-K)+0.60$ & 2.9 & 20.7 & 26.1 \\
\hline$\{R J K\}$ & $R J J K$ & $R K J K$ & RJRK & $1.99(J-K)-1.18^{a}$ & 2.2 & 20.7 & 25.4 \\
\hline$\{I J K\}$ & $I J J K$ & $I K J K$ & $I J I K$ & $1.21(J-K)-0.64^{a, b}$ & 2.2 & 20.7 & 25.4 \\
\hline$\{R J H\}$ & $R J J H$ & RHJH & $R J R H$ & $1.77(J-H)+1.10$ & 2.1 & 20.7 & 25.3 \\
\hline$\{R I J\}$ & $R I I J$ & $R J I J$ & $R I R J$ & $(R-I)>-0.38(I-J)+2.05$ & 1.6 & 20.7 & 24.8 \\
\hline$\{R I K\}$ & RIIK & $R I R K$ & $R K I K$ & $0.22(I-K)+0.39$ & 1.4 & 20.6 & 24.6 \\
\hline$\{R I H\}$ & RIIH & RHIH & RIRH & $0.25(I-H)+0.56$ & 1.2 & 20.5 & 24.4 \\
\hline$\{I H K\}$ & IHHK & $I K H K$ & IHIK & & & & \\
\hline$\{J H K\}$ & JHHK & $J K H K$ & $J H J K$ & & & & \\
\hline$\{I J H\}$ & $I J J H$ & IHJH & $I J I H$ & & & & \\
\hline$\{R J H K\}$ & RJHK & RHJK & RKJH & $4.59(H-K)-1.44^{a}$ & 2.6 & 20.7 & 25.8 \\
\hline$\{I J H K\}$ & IHJK & $I J H K$ & $I K J H$ & $1.78(J-K)-0.72^{a, b}$ & 2.4 & 20.7 & 25.6 \\
\hline$\{R I H K\}$ & $R I H K$ & RHIK & $R K I H$ & $1.80(H-K)-0.59^{a}$ & 2.0 & 20.7 & 25.2 \\
\hline$\{R I J K\}$ & $R I J K$ & $R J I K$ & $R K I J$ & $0.78(J-K)-0.35^{a}$ & 1.8 & 20.7 & 25.0 \\
\hline$\{R I J H\}$ & $R J I H$ & RIJH & RHIJ & $0.65(I-H)+1.20$ & 1.8 & 20.7 & 25.0 \\
\hline
\end{tabular}

${ }^{a}$ Line parallel with reddening vector.

${ }^{b}$ Line runs partly through the colour-colour regions due to complex region shapes.

Table 4. The consistency of the classifications made using different filter configurations are checked against classifications of samples of EROs in the literature. The consistency is grouped according to classification method: (i) the photometric classifications by Smail et al. (2002); (ii) the spectroscopic classifications by Graham \& Dey (1996), Spinrad et al. (1997), Soifer et al. (1999), Afonso et al. (2001), Smith et al. (2001), Frayer et al. (2003) and Saracco et al. (2003); and (iii) the morphologic classifications by Franceschini et al. (1998), Stiavelli et al. (1999), Smith et al. (2002a) and van Dokkum \& Stanford (2003). The consistency is given both as a colour consistency, i.e. the consistency with the separation lines given in Table 3, and a region consistency, i.e. if the sources fall in the proper colour-colour regions. The consistency is given as the number of sources on the correct side of the separation line (or in the correct colour region) over the respective number of sources available.

\begin{tabular}{|c|c|c|c|c|c|c|c|c|}
\hline \multirow[t]{2}{*}{ Filter set } & \multirow[t]{2}{*}{ Configuration } & \multirow[t]{2}{*}{$z_{\max }$} & \multicolumn{6}{|c|}{ Colour (region) consistency for different classification methods } \\
\hline & & & \multicolumn{2}{|c|}{$\begin{array}{l}\text { Smail et al. } 2002 \text { sample } \\
\text { Evolved Dusty }\end{array}$} & \multicolumn{2}{|c|}{$\begin{array}{l}\text { Spectroscopically classified } \\
\text { Evolved Dusty }\end{array}$} & \multicolumn{2}{|c|}{$\begin{array}{l}\text { Morphologically classified } \\
\text { Evolved Dusty }\end{array}$} \\
\hline$\{R H K\}$ & RHHK & 2.9 & $6(6) / 8$ & $11(11) / 15$ & $2(2) / 2$ & $1(1) / 1$ & $1(1) / 1$ & \\
\hline$\{R J K\}$ & RJJK & 2.2 & $9(8) / 9$ & $3\left(1^{a}\right) / 9$ & $4(4) / 4$ & $2(2) / 3$ & $3(3) / 4$ & $0(0) / 10$ \\
\hline$\{I J K\}$ & $I J J K$ & 2.2 & $7(4) / 7$ & $4(3) / 10$ & $3(2) / 3$ & $0\left(0^{a}\right) / 3$ & $20(11) / 21$ & $1\left(1^{a}\right) / 9$ \\
\hline$\{R J H\}$ & $R J J H$ & 2.1 & $4(2) / 5$ & $1(1) / 9$ & $2(2) / 2$ & $0(0) / 1$ & $0(0) / 1$ & \\
\hline$\{R I J\}$ & RIIJ & 1.6 & $5(3) / 7$ & $5(3) / 9$ & $3(1) / 3$ & $1(0) / 2$ & $4(2) / 4$ & $0(0) / 9$ \\
\hline$\{R I K\}$ & RIIK & 1.4 & $3(2) / 10$ & $11(10) / 17$ & $1(1) / 3$ & $1\left(0^{a}\right) / 2$ & $4(4) / 8$ & $9\left(7^{a}\right) / 20$ \\
\hline$\{R I H\}$ & RIIH & 1.2 & $2(0) / 8$ & $9(6) / 15$ & $1(0) / 1$ & $0(0) / 1$ & $0(0) / 1$ & \\
\hline$\{R J H K\}$ & RJHK & 2.6 & $5(5) / 5$ & $7\left(3^{a}\right) / 9$ & $2(2) / 2$ & $1\left(0^{a}\right) / 1$ & $1(1) / 1$ & \\
\hline$\{I J H K\}$ & $I H J K$ & 2.4 & $5(4) / 5$ & $5(3) / 10$ & $1(1) / 1$ & $1(1) / 2$ & $17(11) / 18$ & \\
\hline$\{R I H K\}$ & $R I H K$ & 2.0 & $5(5) / 8$ & $11\left(7^{a}\right) / 15$ & $1(1) / 1$ & $1\left(0^{a}\right) / 1$ & $0(0) / 1$ & \\
\hline$\{R I J K\}$ & $R I J K$ & 1.8 & $5(3) / 7$ & $6(3) / 9$ & $3(3) / 3$ & $2\left(0^{a}\right) / 2$ & $2(2) / 4$ & $4\left(3^{a}\right) / 9$ \\
\hline$\{R I J H\}$ & $R J I H$ & 1.8 & $4(2) / 5$ & $2(0) / 9$ & $1(1) / 1$ & $0(0) / 1$ & $0(0) / 1$ & \\
\hline
\end{tabular}

${ }^{a}$ The region consistency can be improved by using a higher extinction.

To check the performance of the different filter configurations, comparisons are made with existing classifications in the literature. This is shown in Table 4. The consistency of the classifications made here is checked according to classification method: photometric, spectroscopic or morphologic. The photometric consistency check is made against the data in Smail et al. (2002, S02 hereafter). S02 have obtained a sample of
EROs from deep RIzJHK photometry and have also classified the sources by template fitting. The sample is thus divided into two classes where the photometry is consistent with "dusty" or "evolved" galaxies. The spectroscopically classified set is obtained from Graham \& Dey (1996), Spinrad et al. (1997), Soifer et al. (1999), Afonso et al. (2001), Smith et al. (2001), Frayer et al. (2003) and Saracco et al. (2003). The morphologically 


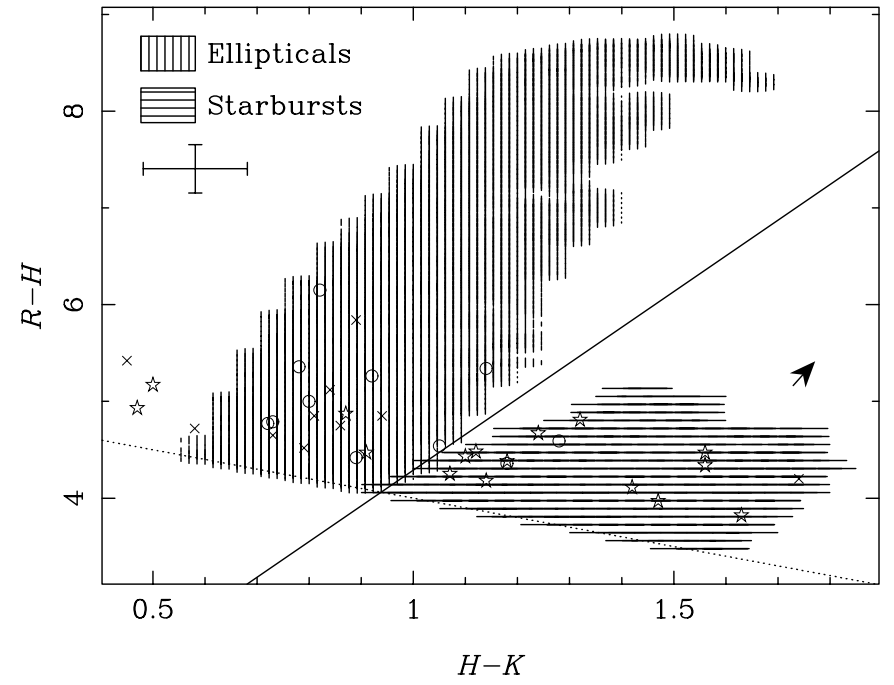

Fig. 7. $R-H$ vs. $H-K$ regions for the Salpeter and Kroupa IMF models for $z \leq 2.9$ having extinctions $E(B-V) \leq 2$, plotted together with classified EROs (references in text and in Table 4). Stars denote dusty galaxies, circles evolved galaxies, and crosses denote the unclassified detections by Smail et al. (2002). The arrow indicates how the starburst region shifts if the colour excess is increased by 0.1 . The error bar shows the typical photometric error of the colours of the EROs.

classified EROs are obtained from Franceschini et al. (1998), Stiavelli et al. (1999), Smith et al. (2002a) and van Dokkum \& Stanford (2003). Smith et al. (2002a) use the classification "Irregular" and "Compact", which, for the consistency check, is simply interpreted as "starburst" and "elliptical" (or "dusty" and "evolved"). This might be too simplistic which would explain the apparently large discrepancy for the morphologically classified starbursts, since the Smith et al. (2002a) sample comprises the majority of these sources.

The RHHK configuration is the best one as it concerns redshift, and it also agrees well with existing classifications. Figure 7 shows the $R H H K$ colour diagram for starbursts and ellipticals up to redshift 2.9. This filter configuration agrees with all the spectroscopically and morphologically classified sources - although the number of sources with $H$ band data is limited - and with the classifications made by S02 for 11 of 15 dusty and 6 of 8 evolved galaxies that have data in these three bands. Furthermore, one of the 11 unclassified S02 sources is found to have the colours of starbursts and 8 (possibly 10) resemble ellipticals. The sources that end up inside the "wrong" colour region can be explained as lower-redshift starbursts having colour excesses between 2 and 3 and ellipticals at redshifts higher than 2.9, respectively. This combination has the property of being a minimal choice of filters, since it only requires one extra filter except for the ERO criterion filters. Figure 1 shows how the $R-K$ colour can select the extremely red elliptical and starburst galaxies, and how they can be distinguished between in the $H$ band.

The RJJK configuration has a smaller redshift coverage, but is also a minimal choice of filters. However, this does not agree with the existing classifications very well, in particular the consistency for the starbursts is bad. In this configuration, a majority of the sources have colours that are consistent with the colours of the model ellipticals. The mismatch also occurs for the $\{I J K\}$ configurations, where additionally the complicated structure of the colour-colour regions makes it difficult to define a clear separation line. The configurations in the $\{I H K\}$, $\{J H K\}$ and $\{I J H\}$ triplets have all very high overlap at low redshifts, which can be attributed to the problem lined out in remark (ii) in Sect. 4.2.1 above. However, as these high overlap regions extend over such considerable redshift space, these filter configurations must be regarded as having a poor discriminating ability.

The best quadruple configuration is $R J H K$. The separation line in this case agrees well with earlier classifications, but a majority of the "dusty" S02 sources fall slightly outside the starburst region. A heavier extinction could justify this, though.

A problem with this kind of method to classify EROs is indicated by the last two columns in Table 3 . These show the $K$ magnitude of the faintest $L^{*}\left(2 \times 10^{10} L_{\odot}\right)$ ERO at the limiting redshift for each colour plane. In general, an $L^{*}$ starburst ERO is 4-6 mag fainter in the $K$ band than an elliptical at $z_{\max }$. This points to a severe difficulty for studies of EROs aimed at disentangling this population: when a study is deep enough to detect "ordinary" starburst galaxies, ellipticals at redshifts far beyond $z_{\max }$ will also be detected. Without redshift information, these high-redshift ellipticals will be included in the sample, and the result will be colour planes with a large amount of confusion. On the other hand, a more shallow survey will tend to detect a majority of ellipticals, which can mislead the conclusions.

In its extension, this problem can affect conclusions drawn from studies aimed at determining the fractions of different galaxy types among the EROs using any of the standard methods. Considering these differences in magnitude of the two populations, it seems plausible that the conclusion that ellipticals dominate the ERO population might just be an effect of this bias. However, a thorough study of the luminosity functions of the galaxy populations constituting the ERO class is required to resolve these implications. The widely different characteristics of the two main candidate populations will presumably make studies aimed at determining the relative fractions of galaxy types among the EROs very sensitive to biases.

\subsubsection{Redder EROs}

The effect of applying higher extinctions is that a greater number of lower-redshift starbursts will be included in the ERO population, some of which will have colours similar to the ellipticals. As mentioned before, the starbursts which are on the correct side of a separation line that is parallel to the reddening vector will not cross the line if the extinction is increased. However, the new ERO candidates among the low-redshift starbursts which previously had $R-K<5$ can, as they are reddened further, appear "from below" and on the wrong side of the separation line, thus ending up in the region of the ellipticals. A conclusion from this, is that the redshift plays a major part in determining which side of the separation line that the starbursts populate. In fact, this appears to be a general effect of the redshift, since if higher-redshift ellipticals are included, these will 


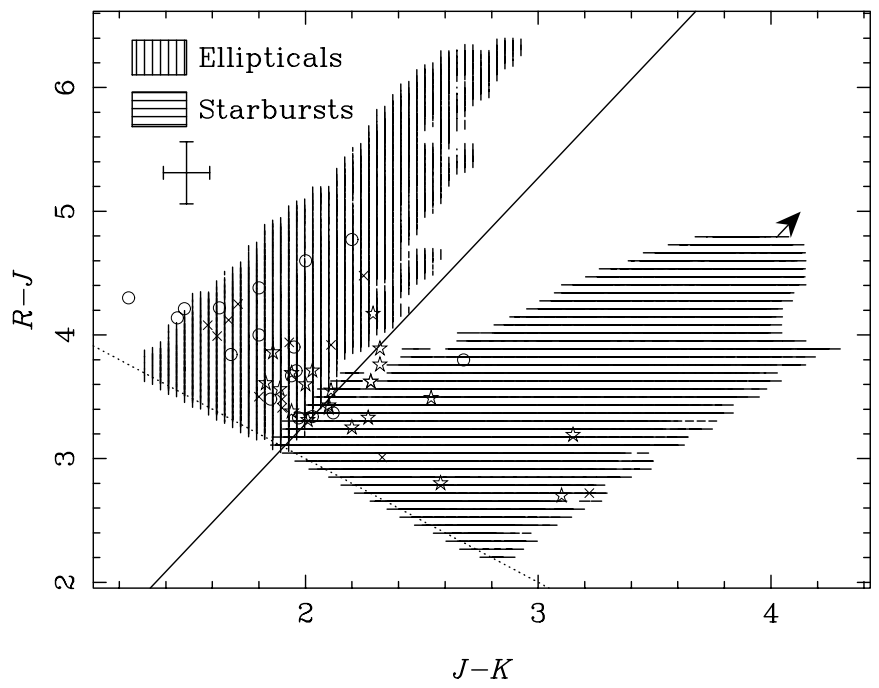

Fig. 8. $R-J$ vs. $J-K$ regions for the Salpeter and Kroupa IMF models for $z \leq 2$ having extinctions $E(B-V) \leq 3$, plotted together with classified EROs (references in text and in Table 4). Stars denote dusty galaxies, circles evolved galaxies, and crosses denote the unclassified detections by Smail et al. (2002). The arrow indicates how the starburst region shifts if the colour excess is increased by 0.1 . The error bar shows the typical photometric error of the colours of the EROs.

move towards the separation line as the redshift is increased, and eventually cross the line.

A higher extinction does in general affect the triplet configurations more than the quadruples. For $E(B-V) \leq 3$, the most stable triplet configuration is $R J J K$, which is valid for $z \leq 2$, although it has a considerable overlap between starbursts at $z \leq 0.5$ and ellipticals at $z \sim 0.8$. Accepting this overlap, the proper separation line is $(R-J)=1.99(J-K)-0.70$; see Fig. 8. The best quadruple configuration when $E(B-V) \leq 3$ is $R J H K$, in which the ellipticals have colours consistent with $(R-J)>0.90(I-K)-0.28$ for $z \leq 1.8$. Note that these two separation lines are parallel with the reddening vector.

The increased overlap at low redshifts caused by high extinction starbursts can be remedied by using a more strict ERO criterion, such as $R-K>6$, which will disentangle the colours by simply de-selecting galaxies, in particular those at $z \lesssim 1$, cf. Figs. 4 and 5. Hence, a stricter criterion will stabilise the discriminating properties of the colour planes, and also in general increase their redshift limits. When the criterion $R-K>6$ is used, the two configurations that have the best redshift coverage are the $R J J K$ (ellipticals have $(R-J)>1.99(J-K)-0.94$ for $z \leq 2.1)$ and the $I J J K((I-J)>1.21(J-K)-0.30$ for $z \leq 2.2)$. Also in these cases, the separation lines are parallel to the reddening vector. The latter configuration will require an ERO selection criterion, though.

The $R H H K$ turns out not to be very stable for an increase of the starburst extinction when using the standard ERO criterion $(R-K>5)$, as lower-redshift starbursts of high extinction are selected and fall in the colour region of the ellipticals. Using the stricter ERO criterion $R-K>6, R H H K$ is the best configuration for $E(B-V) \leq 2$ and is now valid for $z \leq 3.2$. High-redshift ERO ellipticals now force the separation line to the right in Fig. 7; these are consistent with
$(R-H)>4.00(H-K)-0.15$. Pushing the colour excess to higher values shows that $R H H K$ can be used for $E(B-V) \leq 2.4$ when $z \leq 3$. For this high extinction, the position of the separation line is dictated by the starbursts, and is now moved slightly to the left, to $(R-H)>3.69(H-K)+0.77$.

Due to a possible shortcoming of existing spectral evolution synthesis programmes, the modelled SEDs might in fact be too blue. As pointed out by Zackrisson et al. (2001), the pre-main sequence evolution of stars will have a non-negligible effect on the SED during the first tens of Myr for a continuous star formation, a fact which is usually overlooked in existing programmes, such as PÉGASE.2, among others. However, as this phase reddens the SED, this is not a serious problem for this study, because (i) in colour-colour diagrams, such as Figs. 7 and 8 , the starbursts will move up and to the right, and hence away from the loci of the ellipticals, and (ii) since this is a transient effect, the younger starbursts are moved closer to the older starbursts in Fig. 5, which actually narrows the area covered, hence working to decrease the overlap.

\section{Summary and conclusions}

In this paper the colour-colour properties of EROs are investigated. Two types of galaxies, starbursts and ellipticals, have been modelled using the spectral synthesis evolution program PÉGASE.2. The ellipticals are modelled as dust-free galaxies, passively evolving after an instantaneous burst of star formation. The starbursts are modelled as having a constant star formation rate for $100 \mathrm{Myr}$, with dust extinction of various degrees following the Calzetti et al. (2000) obscuration law. Salpeter (1955) and Kroupa (2001, equation (6)) IMFs are used and the metallicities of the galaxies were evolved consistently with age, using initial metallicities from 0.02 up to $2.5 Z_{\odot}$. The colour properties in the 45 colour planes formed from the RIJHK bands are explored up to redshift 5 in a $H_{0}=70 \mathrm{~km} \mathrm{~s}^{-1} \mathrm{Mpc}^{-1}$, $\Omega_{\mathrm{m}}=0.3$ and $\Omega_{\Lambda}=0.7$ cosmology.

The following conclusions can be drawn.

1. Except for the very lowest-metallicity cases, the ellipticals will be EROs at any redshift beyond $\sim 0.6$ in the adopted cosmology. Furthermore, the stellar population has to be formed before $z \sim 1$ and to be consistent with cosmology, an ERO-classified elliptical has to be younger than 7-8 Gyr.

2. The starburst galaxies can be detected as EROs at an extinction of $E(B-V)>1$ at the very highest redshifts. When $E(B-V)=2$ the starbursts have $R-K>5$ at redshifts above $\sim 0.1$.

3. The 30 colour-colour combinations that can be formed from three of the RIJHK filters exhibit the feature that permutations of the filters yield very similar overlap maps. Among the configurations formed from these filter triplets, the ones having the centre waveband as pivot band (i.e. the $H$ band in the $R-H$ vs. $H-K$ configuration) have the best performance as discriminators.

4. Of special interest is the $R-H$ vs. $H-K$ configuration. This provides a minimal choice of filters, as it contains the fundamental ERO criterion, and has a very low degree of overlap between the galaxy populations up to redshifts of 
2.9 for $E(B-V) \leq 2$. Using the alternative ERO criterion $R-K>6$, the $R-H$ vs. $H-K$ configuration works for $E(B-V) \leq 2.4$ and $z \leq 3$.

The results in Sect. 4.2, of which item 4 is a subset, represent the limit of what is achievable with this method of photometric classifications of EROs. Since the chosen star formation histories are extremes (passive evolution versus constant star formation), the separation of the colours of the modelled galaxies is maximised. A galaxy whose star formation history have contributions from both modes of star formation will be more difficult to classify (cf. Pierini et al. 2003). This photometric method is useful for galaxies clearly dominated by either star formation mode, but to securely establish the nature of an ERO other methods have to be applied as well.

Acknowledgements. The authors acknowledge financial support from Vetenskapsrådet (VR; the Swedish Natural Science Council) for this project. S. Bergström is financed through a VR Graduate Student Grant (doktorandtjänst).

\section{References}

Afonso, J., Mobasher, B., Chan, B., \& Cram, L. 2001, ApJ, 559, L101 Bessell, M. S. 1990, PASP, 102, 1181

Bessell, M. S., \& Brett, J. M. 1988, PASP, 100, 1134

Bruzual, G. A., \& Charlot, S. 1991, ApJ, 367, 126

Bruzual, G. A., \& Charlot, S. 1993, ApJ, 405, 538

Calzetti, D., Armus, L., Bohlin, R. C., et al. 2000, ApJ, 533, 682 (C00)

Cimatti, A. 2001, in Deep Fields, 81 [astro-ph/0012057]

Cimatti, A., Andreani, P., Rottgering, H., \& Tilanus, R. 1998, Nature, 392, 895

Cimatti, A., Daddi, E., di Serego Alighieri, S., et al. 1999, A\&A, 352, L45

Cimatti, A., Daddi, E., Mignoli, M., et al. 2002, A\&A, 381, L68

Daddi, E., Cimatti, A., Pozzetti, L., et al. 2000, A\&A, 361, 535

Daddi, E., Cimatti, A., Broadhurst, T., et al. 2002, A\&A, 384, L1

Dey, A., Graham, J. R., Ivison, R. J., et al. 1999, ApJ, 519, 610
Eggen, O. J., Lynden-Bell, D., \& Sandage, A. R. 1962, ApJ, 136, 748

Elston, R., Rieke, G. H., \& Rieke, M. 1988, ApJ, 331, L77

Fioc, M., \& Rocca-Volmerange, B. 1997, A\&A, 326, 950

Fioc, M., \& Rocca-Volmerange, B. 1999 [astro-ph/9912179]

Franceschini, A., Silva, L., Fasano, G., et al. 1998, ApJ, 506, 600

Frayer, D. T., Armus, L., Scoville, N. Z., et al. 2003, AJ, 126, 73

Graham, J. R., \& Dey, A. 1996, ApJ, 471, 720

Kauffmann, G., White, S. D. M., \& Guiderdoni, B. 1993, MNRAS, 264, 201

Kroupa, P. 2001, MNRAS, 322, 231

Larson, R. B. 1975, MNRAS, 173, 671

Mannucci, F., Pozzetti, L., Thompson, D., et al. 2002, MNRAS, 329, L57

Moriondo, G., Cimatti, A., \& Daddi, E. 2000, A\&A, 364, 26

Moustakas, L. A., Casertano, S., Conselice, C., et al. 2003, ApJ, submitted [astro-ph/0309187]

Moustakas, L. A., \& Somerville, R. S. 2002, ApJ, 577, 1

Pierini, D., Maraston, C., Bender, R., \& Witt, A. N. 2003, MNRAS, accepted [astro-ph/0309223]

Pozzetti, L., \& Mannucci, F. 2000, MNRAS, 317, L17 (PM00)

Salpeter, E. E. 1955, ApJ, 121, 161

Saracco, P., Longhetti, M., Severgnini, P., et al. 2003, A\&A, 398, 127

Scalo, J. 1998, in The Stellar Initial Mass Function, 38th Herstmonceux Conf., ASP Conf. Ser., 142, 201

Scodeggio, M., \& Silva, D. R. 2000, A\&A, 359, 953

Smail, I., Owen, F. N., Morrison, G. E., et al. 2002, ApJ, 581, 844 (S02)

Smith, G. P., Treu, T., Ellis, R., et al. 2001, ApJ, 562, 635

Smith, G. P., Smail, I., Kneib, J.-P., et al. 2002a, MNRAS, 330, 1

Smith, G. P., Smail, I., Kneib, J.-P., et al. 2002b, MNRAS, 333, L16

Soifer, B. T., Matthews, K., Neugebauer, G., et al. 1999, AJ, 118, 2065

Spinrad, H., Dey, A., Stern, D., et al. 1997, ApJ, 484, 581

Stiavelli, M., Treu, T., Carollo, C. M., et al. 1999, A\&A, 343, L25

Thompson, D., Beckwith, S. V. W., Fockenbrock, R., et al. 1999, ApJ, 523,100

van Dokkum, P. G., \& Stanford, S. A. 2003, ApJ, 585, 78

White, S. D. M., \& Frenk, C. S. 1991, ApJ, 379, 52

Woosley, S. E., \& Weaver, T. A. 1995, ApJS, 101, 181

Yan, L., \& Thompson, D. 2003, ApJ, 586, 765

Zackrisson, E., Bergvall, N., Olofsson, K., \& Siebert, A. 2001, A\&A, 375,814 